\section{STAB WOUNDS OF THE CHEST}

BY

\author{
TH. SCHRIRE, M.A., M.B.Capetown, F.R.C.S. \\ Surgical Registrar, Groote Schuur Hospital, Capetown
}

It is the universally accepted surgical teaching to explore every penetrating wound to its uttermost depths, arrest the haemorrhage, and so far as possible to remove the damaged tissue. These teachings have stood the test of experience, and one by one the various body cavities have been subjected to these procedures. Chest wounds, however, have remained in the sphere of expectant treatment. Whether this has been due to lack of experience in intrathoracic work or to the comparative freedom from the grosser surgical complications enjoyed by the average chest case I cannot say. I have felt, however, that a test should be made of a series of penetrating wounds of the chest. The opportunity for getting such a series in peacetime seldom arises, but our experience in 26 cases is here presented.

In Capetown one has remarkable opportunities for observing the effects of stab wounds, which within the last twenty years have become extremely common. The Groote Schuur Hospital serves the town and neighbouring suburbs, and about 600 stab cases are treated annually. The majority are of a trivial nature and are dealt with in the out-patient department; but in 1939, for example, on one firm alone, 160 cases were serious enough to require admission and treatment. From such a multitude of cases it is not difficult to get a number large enough to draw some broad conclusions. Accordingly, unless the condition of the patient was so bad as to render any operative intervention an entirely hopeless procedure (and it is noteworthy that as experience increases the proportion of apparently hopeless cases tends to diminish), every patient with a penetrating wound of the chest admitted under my care on Mr. W. L. Gordon's firm was subjected to an exploratory thoracotomy as an emergency measure if seen within twelve hours of the injury.

\section{Diagnosis}

As a rule the diagnosis was obvious: the presence of surgical emphysema, pneumothorax on the same side, and shifting of the apex beat were at all times considered sufficient evidence of penetration. An exploration of the wound in the casualty department, enlarging it if necessary under a local anaesthetic, often showed the site of injury. In several cases omentum or bowel projecting through the intercostal stab has indicated the presence of a thoraco-abdominal wound, and four cases of wounds of the heart and pericardium have been seen these latter form the subject of another communication.

Other Injuries.-Associated with these chest stabs are the usual other injuries sustained in a brawl. Head and limb injuries are not uncommon. Multiple stab wounds are frequent, and as many as six stabs in the back, four on one side and two on the other, have been seen. A large number of our cases show the healed scars, surgical or otherwise, of previous fights.

\section{Treatment}

Pre-operative.-The chest wound is always covered with a biniodide compress held on with strapping in the casualty department. The patients are immediately transferred to the wards and treated for shock. A careful note is made of the apex beat and of the pulse rate, and any sudden displacement of the one or acceleration of the other calls for immediate attention. Several cases have had to be aspirated in the wards for tension pneumothorax; occasionally a blood transfusion is given pre-operatively, but this is not done as a routine. As a rule there is a rapid improvement of the general condition, and within an hour or so the patients are as fit for operation as they will ever be. They are given 1/100 grain of atropine half an hour before going to the table, and they get anti-tetanus and anti-gas serum as a routine.

Anaesthetic.-The anaesthetic of choice would undoubtedly be an intratracheal one of gas and oxygen, but as these cases usually occur at night, during the weekends, we have had to rely upon housemen whose experience in this direction is limited. Accordingly, the simplest anaesthetic is used, and one can get on very well with C.2E.3 induction, followed by ether given through an airway by a Shipway apparatus. This is simple and, in our experience, safe. Positive pressure can be obtained in a crude sort of way by increasing the oxygen flow and partially blocking the airway. It works very well, and the patient's condition seldom gives cause for anxiety. Local anaesthesia would be more often used if the patients were more co-operative and less drunk; as things are, we are seldom able to give it a trial.

In the majority of cases the patient lies on his good side with the upper arm in an arm rest. Stabs near the sternum are best treated with the patient lying on his back. Skin preparation is often done on the theatre table. The skin is cleansed with spirit soap; this is washed off with spirit, and ether is then poured over. Carbolic 1 in 20 is used for all traumatic cases, and is liberally applied.

Incision.-The edges of the original skin wound are excised and the incision is prolonged in the interspace penetrated. To facilitate matters and to speed up the procedure a very generous incision is always employedseldom less than ten inches in length, and often longer. Should it be necessary to enlarge the incision, ribs and interspaces (the latter between two ligatures) are freely divided in the direction required. Anteriorly, the cartilages above and below are divided with a knife for stabs in this situation. The incisions have healed firmly and there have been no difficulties or complications associated with them. Where the lower six interspaces have been cut across the resultant paralysis of the upper part of the rectus abdominis has not been commented upon and has caused no symptoms.

To divide the muscles two fingers are inserted in the fascial plane superficial to the ribs, and the superficial musculature can be divided between these fingers. In this way haemorrhage is controlled and vessels may be caught before they are cut. This is a very rapid way of exposing the chest wall. The vessels are now tied off and the penetrated interspace is chosen for incision.

The intercostal neurovascular bundle is now ligated; the bundle is underpinned with two sutures of chromic catgut on a curved needle and divided between the sutures to make sure that all is secure. This procedure is performed after the rib has been resected, to prevent the waste of time that always occurs if one attempts to catch the intercostal artery lying deep in its bony groove. The freeing of the rib can be rapidly performed with a little practice, especially if it is remembered that, owing to the direction of the fibres of the external intercostal muscle, the superior border is easiest cleaned working from behind forwards and the inferior border from before backwards. An inch of rib near its angle is removed for this purpose.

In anterior incisions it is not necessary to excise a portion of rib because the interspaces are much wider and the vessels do not lie close under the rib margin. The blood supply, too, comes from the internal mammary artery, and time is wasted by tying the intercostal bundle. 
Division of the costal cartilages above and below suffices for retraction.

The interspace incision is now practically bloodless. After the thorax is opened a rib-spreader is introduced. Until a Tudor Edwards double rib-spreader was obtained eighteen months ago, a de Quervain retractor was used with complete satisfaction; the double retractor gives a rather better exposure, but any powerful self-retaining instrument will do. With the chest wall open any procedure can now be undertaken. The blood in the pleura is evacuated by mopping. We have found the "sucker" to be a.nuisance; if weak it does not break up the clots, and if strong the lung is constantly being sucked in. Large moist swabs are used, and only one at a time is put into the pleura.

With the blood cleaned out it is easy to see where the haemorrhage comes from, and this is now dealt with. The lung is grasped with lung forceps and lifted into the wound. All the surfaces of each lobe are examined, and penetrating wounds are sutured with continuous No. 0 chromic catgut on a curved intestinal needle. The pleural surfaces usually come easily into apposition; if not, one or two extra sutures bring them together. Bleeding from the lung is checked by passing the sutures deeply, but it is seldom necessary to do this. Theoretically, the less lung tissue is rendered ischaemic the better. Adhesions, owing to the frequent incidence of tubercle, have often been encountered. They are not broken down, and care is taken to avoid injuring them. They help materially in stabilizing the mediastinum.

The diaphragm is stitched with two layers of No. 2 chromic catgut, and if necessary the parietal wound can be extended across the upper part of the rectus abdominis, converting the thoracic into a thoraco-abdominal wound. A full exposure of the abdomen is then obtainable. Before stitching the diaphragm it is advisable to crush the phrenic nerve as it lies on the pericardium, to steady the movements and aid healing. Wounds of the heart are treated by suture with chromic catgut No. 1 or 2 . Four cases have been encountered, of which only one falls into this series. The pericardium is left widely open, and is allowed to drain into the left or right pleura. No separate drain is used for the pericardium. No wounds of the great vessels have been seen. One case in which there was a wound in the pulmonary artery was, however, reported by Whitaker (1939). It was successfully stitched with three sutures of chromic catgut. Most of the intrapleural haemorrhage comes from the intercostal vessels. The artery, owing to its position in the subcostal groove, is apparently unable to retract efficiently and keeps on bleeding for a long while.

Before the chest is closed a No. 14 self-retaining catheter is put in through a separate stab wound low down in the posterior axillary line. This is stitched in place with a silkworm-gut suture, which is tied off. Another suture is now put across the stab wound and left loose, to be tied when the tube is removed.

Closure is easily effected by the insertion of three or more pericostal sutures which encircle the rib above and below. These sutures pass close to the upper border of the upper rib and to the lower part of the interspace below in order to avoid the intercostal arteries. The ribs are approximated and the pericostal sutures are tied; the superficial muscles are now closed in two layers with a running No. 2 chromic catgut suture, and the skin with interrupted silkworm-gut. It takes too long to suture the cut intercostal muscles, and the sutures tend to tear out; the wounds have done well without this procedure. The patient is returned to bed and the drainage tube is led under water. $\mathrm{He}$ is sat up as soon as he recovers from the anaesthetic.

\section{After-treatment}

The patient is nursed in the Fowler position and is given inhalations of $\mathrm{CO}_{2}$ for two or three minutes every halfhour for the first twenty-four hours. In addition we have been giving full doses of sulphonamide for the first few days. Patients get 1 gramme four times a day for the first two days, 1 gramme three times a day for the next two days, and then 0.5 gramme three times a day for the last two days. We think this has had a considerable effect in reducing the incidence of infection. The thoracotomy wound as a rule heals by first intention. The drainage wound takes a little longer to heal. The tube is removed after thirty-six hours, during the first twenty-four of which about 8 to $10 \mathrm{oz}$. of blood-stained fluid is discharged ; during the last twelve hours there is practically no discharge. Pain is seldom noted after the drainage tube is removed. If there is pain morphine is given freely.

\section{Discussion}

Our aim has been to get the lung re-expanded as soon as possible. Theoretically, this is desirable, because a pleural space partly filled with blood and potentially infected is an ideal situation for invading organisms. The smaller the pleural space the smaller will be the empyema should an infection occur, and the sooner will this heal. There is no worse case than the so-called "total empyema," where the lung has fallen completely away from the chest wall. It takes months to heal and there is considerable pleural scarring. Many of these cases of total empyema will require thoracoplasties for a complete closure.

It is therefore essential to find and stitch the lung wounds so that a negative pressure can be maintained in the pleura to pull the lung out. An open wound in the lung will allow air to leak into the pleura and the negative pressure is lost. In addition the leak in the lung may become valvular and a tension pneumothorax may occur. So long as the tube is in situ and patent everything is safe, but if the tube were to get blocked the condition of the patient would rapidly become serious. We have not seen a tension pneumothorax develop after suture in our series, but we used a wide self-retaining tube to be on the safe side. It is also essential to keep the lungs fully aerated and not to allow the bronchi to become blocked with secretion or blood. Coughing is encouraged and the $\mathrm{CO}_{2}$ inhalations compel deep inspiration and help to loosen any plugs.

\section{Results}

In all, 17 cases were treated by the above method. Several of them are of interest. One patient, a male aged 18 , showed a low-grade pyrexia for several weeks. Radiographs revealed shadows in the good lung which were interpreted as pneumonia. Finally, tuberculosis bacilli were found in the sputum; his disease had apparently become reactivated by his injury, and he was transferred to a tuberculosis sanatorium. A second case, that of a man aged 37, was treated for another firm. The tube was inadvertently left open in his dressing and not put under water. When seen forty-eight hours later it was already too late: the lung had retracted, the pleura became infected, and a total empyema which required strong suction drainage for three months resulted. In the only fatal case in the series the patient, a man aged 35 , had been injured eight hours previously, and in addition to his chest wound had had his left brachial artery and median nerve completely cut across. He lay in the road for four hours and was found unconscious in a pool of blood. Two transfusions allowed him to revive to a sufficient extent to be operated upon, but in spite of another transfusion he died eighteen hours later. The post-mortem examina- 
tion showed that the lung and other wounds were firmly closed, and death was attributed to "secondary shock." In another case the diaphragm, spleen, and right ventricle were injured, the lung being intact. Suture of the ventricle and of the diaphragm was done and primary union occurred.

For comparison 9 cases of penetrating wounds of the chest were treated conservatively. They were not operated upon, either because they came in after twelve hours or because they refused operation. Their average stay in hospital was $11 \frac{1}{2}$ days, as against $16 \frac{1}{2}$ days for the operated cases, excluding two cases in which outside factors influenced their stay. The comparison is not absolutely fair because only the less serious cases are included in the second group; the serious cases are admitted sooner and do not refuse operative treatment.

\section{Summary and Conclusions}

17 consecutive cases of penetrating stab wounds of the chest were treated by operation. There was 1 death.

The average stay in hospital of 14 of these was $16 \frac{1}{2}$ days.

A series of 9 less serious similar stabs treated conservatively had an average of $11 \frac{1}{2}$ days.

The general impression obtained is that operation is worth while. The cases are much less worrying postoperatively: aspirations and repeated pulse observations are not required. All that is necessary is for a nurse to walk down the ward and look at the bottles under the beds. The patients move about freely in their beds, and after the drainage tubes are removed they do not complain of any pain. There is much less pain than after a laparotomy, and we have not come across any of the postoperative complications that occur in the latter.

I would like to express my thanks to my chief, $\mathrm{Mr}$. W. Lennox Gordon, F.R.C.S., for his courtesy in allowing me the use of his beds for treating these cases and for his kindly interest in the work.

REFERENCE

Whitaker, J. C. (1939). J. thorac. Surg., 9, 106.

\section{ATROPINE POISONING DESCRIPTION OF AN UNUSUAL CASE}

BY

\section{A. BARHAM CARTER, M.B., M.R.C.P., D.P.M. \\ Physician to the Central Middlesex County Hospital, London}

Cases of atropine poisoning either with unusual features or occurring in epidemic form were frequently reported in the literature up to 1921 . Since then very few descriptions of this condition have appeared, either because it is occurring less often or because cases of unusual interest are fewer. The following case presents sufficient uncommon features to merit a brief report.

\section{Case History}

The patient, a previously healthy man aged 73 , was admitted to the Central Middlesex County Hospital at 8.30 p.m. on February 4, 1940. The story was that at 11 o'clock the previous night he had reached up to a shelf for a bottle of cough mixture, and by mistake had taken down a 1-oz. bottle full of linimentum belladonnae. This he emptied of all but three drachms at a single draught. He noticed an unusual taste and a burning sensation in his mouth, and realized his mistake. He was not unduly perturbed by this and got into bed and went to sleep immediately. At 7.30 the next morning his wife was awakented by his convulsions in bed, and, becoming alarmed, she summoned a doctor, who sent the patient into hospital at once. No vomiting had occurred and no treatment had been given.

On admission, ten hours after taking the liniment, the patient was unconscious, with flushed face, stertorous breathing, and slight excoriations on the lips. His tongue and mouth were dry and leathery. The pupils were fully dilated and without reaction to light. All his limbs showed increased resistance to passive movement, and frequent convulsive movements of legs and arms occurred equally on both sides. Occasionally there was bilateral twitching of the lower face. All the tendon reflexes were brisk and equal, and an extensor plantar response was obtained on the left side. The corneal reflex was present, but the conjunctival reflex was absent. The temperature was $99.6^{\circ}$ and the respirations 26 . The cardiovascular system showed a pulse of 120 , irregular, with extrasystoles. The blood pressure was $135 / 90$. Heart sounds were poorly heard. The bladder was distended and $35 \mathrm{oz}$. of urine was subsequently withdrawn.

Morphine, 1/4 grain, was given immediately, with physostigmine, 1/50 grain. The stomach was empty, but free lavage with potassium permanganate 1 in 5,000 was carried out and half a pint of black coffee was left behind. The convulsive movements continued and the patient's condition was unchanged an hour later, except that restlessness was, if anything, increasing. Lumbar puncture was performed at 11.30 a.m. and $30 \mathrm{c} . \mathrm{cm}$. of clear fluid withdrawn, the pressure falling from $200 \mathrm{~mm}$. of water to 70 . Shortly after this it was possible to rouse the patient. He was resistive and disoriented, mistaking the hospital and his nurses for his home and family. There was no direct evidence of hallucinations.

Morphine, 1/4 grain, and physostigmine, 1/50 grain, were again given at 12 noon, and the convulsive movements grew less severe. Perspiration now became profuse and he began to take fluids well. The face was still flushed, the mouth dry, the eyes sparkling, and the pupils dilated and fixed. His speech was ataxic and slurred, with rambling incoherent phrases, and he began to show seemingly purposeful movements of his hands and arms. He tried to get out of bed, and required more attention. His pulse was stronger, and the blood pressure $150 / 80$, but rhythm was unchanged. He could be fed with fluids without much difficulty.

At 5 p.m., six hours after admission, he was still semicomatose, with fixed pupils, flushed face, and profuse sweating; he vomited $3 \mathrm{oz}$. of bile-stained fluid. The reflexes were brisk, the plantars flexor, the limbs less spastic, and the convulsive movements had ceased. He was restless and voluble, and seemed to be hallucinated, picking at the bedclothes. Morphine, 1/6 grain, was given. At 8 p.m. he was more rational, asking where he was: he said he was very thirsty and had burning sensations in his feet. He was sweating profusely all over, and stated that everything he looked at was blurred. He went to sleep at 11 p.m., still sweating.

Next day he was mildly excited and elated, voluble but quite rational. He was unable to give any history of the poisoning. He complained of thirst, burning of throat, unsteadiness of limbs, and headache. He still could not see distinctly. He took fluids well and had full control of his sphincters. The only abnormal physical signs were fixed dilated pupils and a persistently raised pulse rate of 120 . On the third day after admission he gave a clear rational account of the accident and showed a mild euphoria, tending to laugh about the whole affair. He was quite normal the next day; his temperature and pulse had settled, but his pupils were still widely dilated and showed only the faintest reaction to light.

The patient was discharged fully recovered eleven days after admission, with pupils still larger than normal and reacting poorly to light: there were no other abnormal signs or symptoms. When seen again two months later he was in perfect health.

\section{Discussion}

Most of the reported cases of atropine poisoning in advanced age with recovery are characterized by small dosage, and illustrate the accepted teaching that susceptibility to atropine increases with age. Moorhead (1919) describes two cases of severe poisoning, one in a man aged 84 and the other in a woman aged 75 , both caused 